\title{
Study at non-destructive testing technology based on vibration characteristics of the steel rod with crack
}

\author{
Hongchun Sun ${ }^{1, a}$, Lina Wang ${ }^{1, b}$ and Yize Liu $^{2, c}$ \\ ${ }^{1}$ School of Mechanical Engineering and Automation, Northeastern University, Shenyang, China \\ ${ }^{2}$ Nuclear Power Institute of China,Chengdu,China \\ ahchsun@mail.neu.edu.cn, ' Inw_neu@163.coml, ' $y i z e 130 @ 126 . c o m$
}

Keywords: non-destructive testing, vibration characteristics, steel rod, crack, wavelet modulus maxima.

\begin{abstract}
Non-destructive testing technology of a steel bar with crack are analyzed and discussed based on vibration characteristics method. The natural frequencies, mode shapes and modal curvature modal for crack of steel bar were analyzed using the method of finite element analysis. Thus it is concluded that the modal curvature mode can be better characterization of damage properties. In order to determine crack depth, the mathematical expressions between damage factor and crack depth are constructed through wavelet analysis of curvature mode. The results for the selection of dynamic damage identification index and non-destructive testing of member bar structures for engineering structure have engineering application value.
\end{abstract}

\section{Introduction}

Steel rod is widely used in bridges, buildings, hoisting machinery, aircraft structure and the structure of the sucker rod pumping unit. Because of its manufacturing, processing and the complexity of the work environment, it will make the new steel rods or steel bar in service to cause a certain degree of crack damage, the emergence and extensions of structural cracks is the first performance of structural damage[1]. Ultrasonic, electromagnetic, radiation, eddy current, heat methods to detect cracks are complex, detect using a long time, expensive high precision and signal extraction difficulty. Thus, the crack damage identification method based on vibration characteristics was put forward. The method will be able to reflect changes in structural damage amount as damage identification factor(damage sensitive indicators), determine the location and degree of structural damage by observing the changes in these factors. It has the advantages of non destructive, convenient, fast and cheap, extracting easily vibration signal at the same time. Because of advantages[2] that it can detect the structure of not be able to directly test location damage, it is widely used. The article used different depth of crack of steel bar as the research object, and got the vibration characteristic of the different crack location and degree using a mathematical model, which can be a way to detect cracks in the steel rod. Wavelet modulus maxima of curvature mode was used to characterize crack damage in this paper, and the relationship between damage factor and crack depth was established, then it can locate damage efficiently and determine crack depth.

\section{Establishment of the steel bar model}

Natural frequencies, vibration modal and curvature modal of structure were got with using the finite element analysis. The sensitive parameters to affect the crack damage were got by using analysis and comparison. The steel bars with different depth cracks were analyzed by finite element analysis software, the parameters of rod material are shown as followed : Elastic modulus E=2.1e5MPa, Poisson ratio $\varepsilon=0.3$, Density $\rho=7.85 \mathrm{e}-6 \mathrm{~kg} / \mathrm{mm} 3$. The structural parameters of rod are that Diameter $\mathrm{d}=8 \mathrm{~mm}$, Length of the rod $\mathrm{L}=484 \mathrm{~mm}$. The depth of crack was expressed by depth-diameter ratio $\mathrm{h} / \mathrm{d}$, seven kinds of crack bar were analyzed. $\mathrm{h} / \mathrm{d}=0,0.050,0.075,0.100,0.125,0.150,0.175$. In the analysis, the rod body that was clamped on both ends was selected as the research object, each of the two sides 
was fixed, its length was $32 \mathrm{~mm}$, the effective length of the rod $\mathrm{l}=420 \mathrm{~mm}$. The rectangular notch that was at the left end $105 \mathrm{~mm}$ of rod was simulated crack. Because the damage degree of rod was very small when the width of the crack changes [3], width values of the crack were $1 \mathrm{~mm}$ in order to theoretical studies and sample processing. The model is shown in Fig.1.

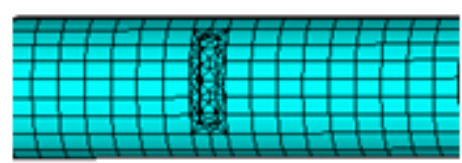

Fig. 1 Crack model

\section{Vibration characteristics of steel bars with the crack}

Analysis of natural frequency. When structural damage occurs, it will affect the modal parameters of structure to change, such as mode shapes, frequencies and so on. Seven kinds of rods with different depth of crack damage were analyzed for the natural frequency, the frequency values of which were shown in Table 1, where "-" is vibration in the horizontal direction. "上" is vibration in the vertical direction.

Table1 Natural frequencies of bars with different damage degrees $(\mathrm{Hz})$

\begin{tabular}{|c|c|c|c|c|c|c|}
\hline $\mathrm{h} / \mathrm{d}$ & $1 \mathrm{st}(-)$ & $1 \mathrm{st}(\perp)$ & 2nd(一) & 2nd( $(\stackrel{\perp}{)}$ & $3 \mathrm{rd}(-)$ & $3 r d(\perp)$ \\
\hline 0.000 & 199.67 & 203.54 & 549.86 & 559.89 & 1074.3 & 1094.5 \\
\hline 0.050 & 199.67 & 203.54 & 549.41 & 559.78 & 1074.1 & 1094.2 \\
\hline 0.075 & 199.60 & 203.38 & 549.31 & 559.63 & 1073.5 & 1093.9 \\
\hline 0.100 & 199.62 & 203.53 & 549.20 & 559.27 & 1073.1 & 1092.0 \\
\hline 0.125 & 199.18 & 203.50 & 549.06 & 559.14 & 1073.8 & 1092.9 \\
\hline 0.150 & 199.38 & 203.38 & 549.40 & 558.78 & 1073.3 & 1092.2 \\
\hline 0.175 & 199.29 & 203.46 & 549.37 & 557.35 & 1073.4 & 1091.3 \\
\hline
\end{tabular}

When small cracks appear in the rod, the natural frequency of the rod is slightly lower (as shown in Table 1). The result indicates that the change of frequency can identify the presence of damage, but it cannot identify the specific location of the damage. Table 1 shows that there is no correlation between the change of frequency and the change in depth of the crack. Therefore, the frequency cannot be used as the sensitive indicator that identifies the location and extent of damage.

Vibration mode analysis. Figs.2-4 show modal shapes from 1st to 3rd order of the rod with $\mathrm{h} / \mathrm{d}=$ 0.125 depth crack. The vibration modal shapes are very smooth, which can't be effectively reflected the presence of crack damage, so modal shape theoretical analysis is not sensitive to damage caused by small cracks.

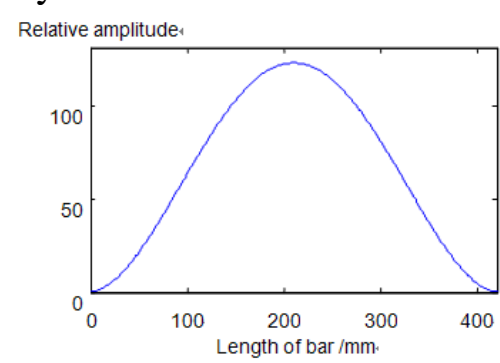

Fig.2 the first order modal shape

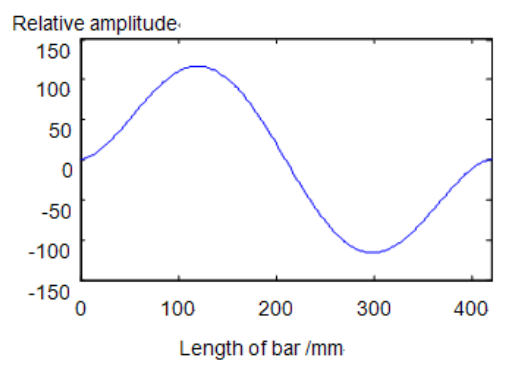

Fig.3 the second order modal shape

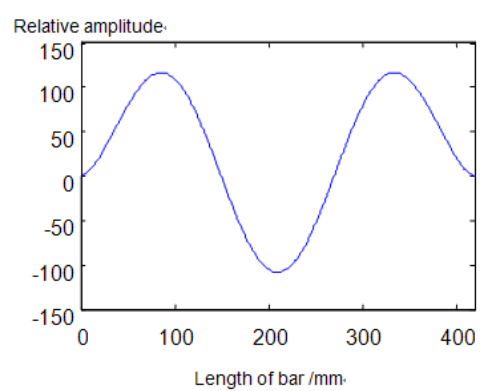

Fig.4 the third order modal shape

Curvature mode shapes analysis. As theoretical knowledge of material mechanics is known[4], when the structure under load can bend. Bending curvature of the structure can be expressed as:

$\frac{1}{\rho(x, t)}=\frac{M(x, t)}{E I(x)}$ 
Where $\rho(x, t)$ is curvature radius when the medial axis of structure is bended, $1 / \rho(x, t)$ is curvature, $M(x, t)$ is the moment of structure and $E I(\mathrm{x})$ is the flexural rigidity of structure. When there is damage in the local structure, the local flexural rigidity of damage location will fall, which can increase the curvature of the location and the value of curvature function at the damage location, therefore the change of curvature can identify structural damage [5].

In the actual analysis, the curvature mode shapes of engineering structures are difficult to measure directly, which often uses the second-order central difference method to approximatively calculate the curvature mode shapes of engineering structures [6]. Curvature mode can be approximatively expressed as:

$$
\ddot{\phi}_{i k} \approx \frac{\phi_{i(k+1)}-2 \phi_{i k}+\phi_{i(k-1)}}{\lambda^{2}}
$$

Where $\ddot{\phi}_{i k}$ is the amplitude of i-order vibration mode, $k$ is the number of measure point in the experiment and $\lambda$ is the distance between two adjacent points .

Therefore, in order to get the curvature mode of structure in the project, the modal of the structure can be got by modal analysis, and then the curvature mode shape of the structure can be got by Formula (2).

The modal mode shape of 2.2 is done curvature mode shape analysis, the diagram of curvature the mode shape can be got. Compared to Figs.2-4, Figs.5-7 appear obvious mutations with crack, and mutations are in exactly corresponding to the crack damage location. Therefore, the curvature modal shape is very sensitive to small crack damage of rods body, it can be initially used as a parameter indicators to measure crack damage. Considering noise and other factors interference in the modal experiment, higher order mode shape is not easy to measure or whose error is relatively large, so the second-order curvature mode shapes are selected as an indicator.

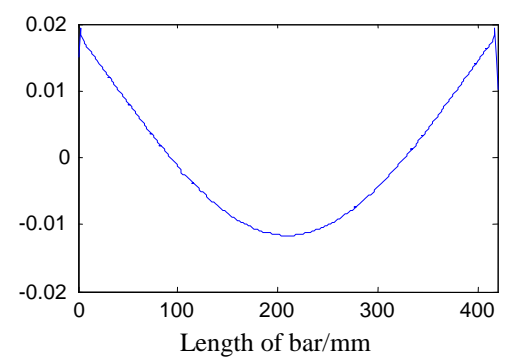

Fig.5 the first curvature mode shapes

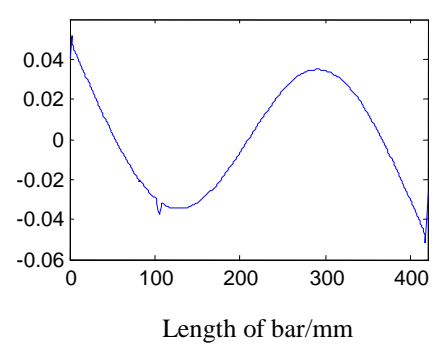

Fig.6 the second curvature mode shapes

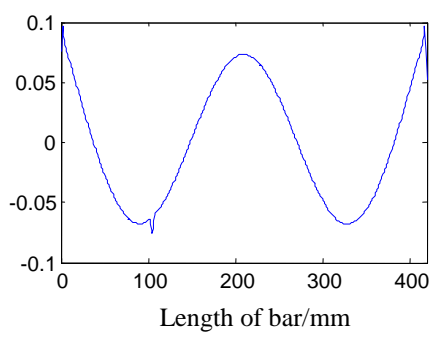

Fig. 7 the third curvature mode shapes

\section{Crack damage determination based on wavelet modulus maxima}

To further ascertain crack location and quantify crack damage, wavelet multi-scale analysis is conducted on the injury information contained in the second-order curvature modal shape above, wavelet coefficient figure can be got. Now taking the steel bar contained a crack whose $\mathrm{h} / \mathrm{d}$ is 0.125 for example, as shown in Fig.8.

The point $\mathrm{A}$ is the wavelet modulus maximum in Fig. 8, and the point $\mathrm{A}$ which is at the position of $105 \mathrm{~mm}$, exactly matches with the preset crack location, so the position of single crack damage in steel bar can be accurately achieved through wavelet modulus maximum points in wavelet coefficient figure. 


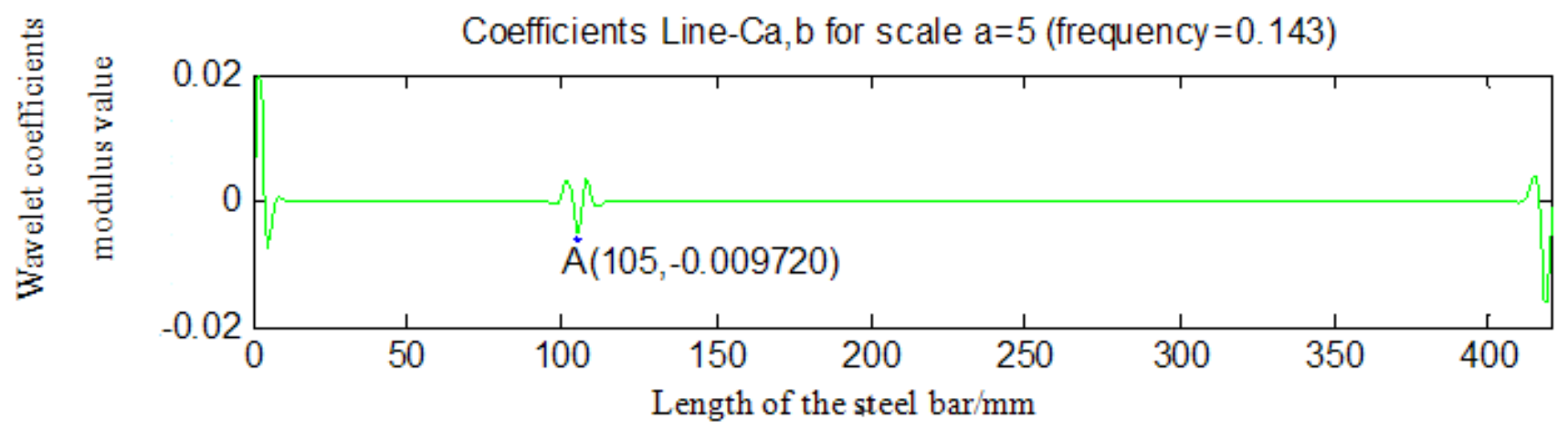

Fig. 8 The fifth scale wavelet coefficients figure

After the curvature modal wavelet analysis for steel bars with different depth cracks, multi-scale wavelet analysis is conducted as well, the wavelet modulus maxima of the point A we got are as shown in Table 2.

Table 2 the wavelet transform modulus maxima of 1 to 5 scale for different depth cracks $\left|W_{s} f(x)\right|$

\begin{tabular}{l|cccccc}
\hline & & & & & \\
\end{tabular}

the formula $\log _{2}\left|W_{s} f(x)\right|=\log _{2} A+(a+1 / 2) \log _{2} s$, Lipschitz index $a$ of function $f(t)$ at $x_{0}$ point is the maximum slope subtract $1 / 2$, and the maximum slope is obtained when $\log _{2}\left|W_{s} f(x)\right|$ as the function of $\log _{2} s$ along the maximum curve converging to $x_{0}$ point. Therefore, we obtain the Lipschitz index $a$ value for different crack depth as shown in Table 3.

Table3 Lipschitz index $a$ value for different crack depth

\begin{tabular}{|c|c|c|c|c|c|c|}
\hline Damage $h / d$ & 0.050 & 0.075 & 0.100 & 0.125 & 0.150 & 0.175 \\
\hline Lipschitz $a$ & 1.4186 & 1.3337 & 1.2351 & 1.2266 & 1.2034 & 1.1770 \\
\hline
\end{tabular}

As shown from Table 3, when the damage level $h / d$ is smaller, the crack depth is smaller, the value of Lipschitz index $a$ is larger, on the contrary, when $h / d$ is larger, $a$ is smaller, which is consistent with the view raised by JC Hong ${ }^{[9]}$, their fitting curve is shown in Fig.9.

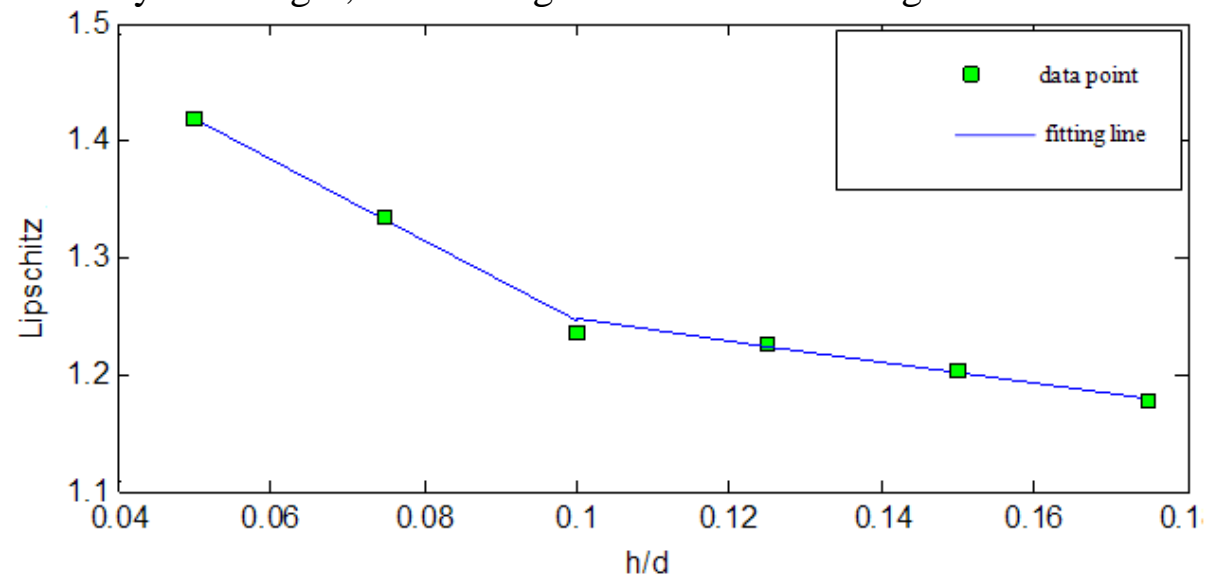

Fig. 9 The relationship graph between the Lipschitz value and crack depth of the steel bar $\mathrm{h} / \mathrm{d}=0.1$ is the boundary of fitting equation in Fig. 9 since the $\mathrm{h} / \mathrm{d}$ of cracks studied in this paper is between 0.005 and 0.175 , it is divided into two parts:

When $0.005 \leq h / d \leq 0.100$,fitting equation is $y_{1}=-3.47 x+1.5927$, fitting coefficient of determination $R_{1}=0.9998$ the equation is valid; 
When $0.100 \leq h / d \leq 0.175$,fitting equation is $y_{2}=-0.91 x+1.3381$, fitting coefficient of determination $R_{2}=0.9940$ the equation is valid;

Which $x=h / d, y$ is the corresponding value of Lipschitz .

\section{Conclusion}

The modal parameters of frequency, mode shapes, modal shape and so on are obtained by the modal analysis calculation of steel bar. By sensitivity analysis, the curvature mode has obvious characterization to detect damage. Choosing Lipschitz value as the crack damage indicator, the greater the crack depth is, the smaller the Lipschitz value is, constructing the mathematical model of depth to diameter ratio of cracks and Lipschitz, and it can well positioning and quantify crack in steel bars.

\section{Acknowledgements}

This work was supported by the National Natural Science Foundation of China (Grant No.51335003).

\section{References}

[1] J.F. Zhang and Y.D. Zhao: Development status and prospect of damage vibration diagnosis of structure crack. Journal of Vibration and Shock, Vol.21(4) (2002), 24-28.

[2] W.C. Gao, W.Liu and J.X. Zou: Damage detection method review based on the change of structural vibration parameters. Journal of Vibration and Shock Vol. 23(4) ( 2004), 1-7.

[3] Y.C.Deng ,in:The Analysis Research of Rod Crack Identification Method Based on Modal Analysis research, Northeastern University- Shenyang(2012).

[4] H.W. Liu,in: Mechanics of Materials, Higher Education Press- Beijing (2004).

[5] W.L. Huang,in:Study on modal identification and damage diagnosis of a model arch bridge, Beijing Jiaotong University -Beijing(2011).

[6] S.Y. Ni,in:Research the vibration properities of wood plastic composite and its inner defects detection based on modal analysis, Northeast Forestry University- Harbin(2011).

[7]Mohammad Ameen Al-Shudeifat, B.S.and M.S.in:New techniques for order reduction and damage detection in structural and rotor dynamic systems. New Mexico State University -New Mexico(2010).

[8]Lin Baolong, Application of Wavelet Transform on Structural Damage Identification ,: Zhejiang University- Zhejiang(2006).

[9] J.C.Hong, Y. Y. Kim, H.C. Lee, Y. W. Lee. Damage detection using the Lipschitz exponent estimated by the wavelet transform: applications to vibration modes of a beam. International Journal of Solids and Structures, Vol.39 (7) (2002) 1803-1816. 